\title{
Study of the Global Performance Index of Parallel Mechanism
}

\author{
Qi-Kai YU ${ }^{1,2, a}$, You-Peng YOU ${ }^{1}$, Jiang-Yi HAN ${ }^{3}$
}

${ }^{1}$ Jiangsu Key Laboratory of Precision and Micro-Manufacturing Technology, Nanjing University of Aeronautics and Astronautics, Nanjing, China

${ }^{2}$ Mechanical Engineering department, Nanjing Polytechnic Institute, Nanjing, China

${ }^{3}$ School of Automotive and Traffic Engineering, Jiangsu University, Zhenjiang, China

ayuqikai@163.com

Keywords: Parallel Mechanism, Jacobi Matrix, Global Performance Index.

\begin{abstract}
There are some one-sided in the robot performance index of the current robot. This paper applies delta parallel mechanism to analyze the condition number index and global performance index (GPI). An improved global motion performance volatility index is also introduced according to the deficiency of GPI. The objective optimization function is established and two sets of satisfactory optimization results were obtained. The global performance volatility was later used to select the optimum result in a given space. Finally, Volatility index were studied and successful implementations were conducted on manipulators.
\end{abstract}

\section{Introduction}

The performance index of the robot is considered as one of the important basis for the selection of hand controller. Appropriate selection of the robot performance evaluation index has been always the source of attentions by many domestic and international researchers. As a micro-operation hand controller requires high precision, the parallel mechanisms is proposed as a suitable candidate with many advantages including high stiffness, high velocity, compactness, high load or weight ratio, and low moving inertia. Large space, fast and high precision characteristic are the key elements to select the parallel robot with strong bearing capacity.

Salisbury ${ }^{[1]}$ defines the isotropic index and the condition number of Jacobian matrix, but it describes a local property of robot motion. To overcome the lacks, Gosselin and Angeles ${ }^{[2-3]}$ proposed a global performance index (GCI). The condition number index and GCI are usually applied in the optimum design of parallel manipulators ${ }^{[4-6]}$, but the condition number index and GCI are not very comprehensive. It is merely a reflection of the robot in the working space average movement performance, and not of the performance of the robot in the working space fluctuation range.

This paper is based on the Delta mechanism as a manipulator which is used to analysis the mechanism parameters change impact on the fluctuation of GCI. Although the Delta mechanism has been widely used in industrial and research works, but it is still recommended to optimize manipulator mechanism with respect to its area of application.

The Delta mechanism (see Fig.1) is a kind of parallel mechanism ${ }^{[7]}$, which comprises of moving platform, fixed flat and three branched chains. Each branch chain consists of upper arm and lower arm. The forward and inverse positions mechanism has been comprehensively studied in ${ }^{[8-10]}$. 

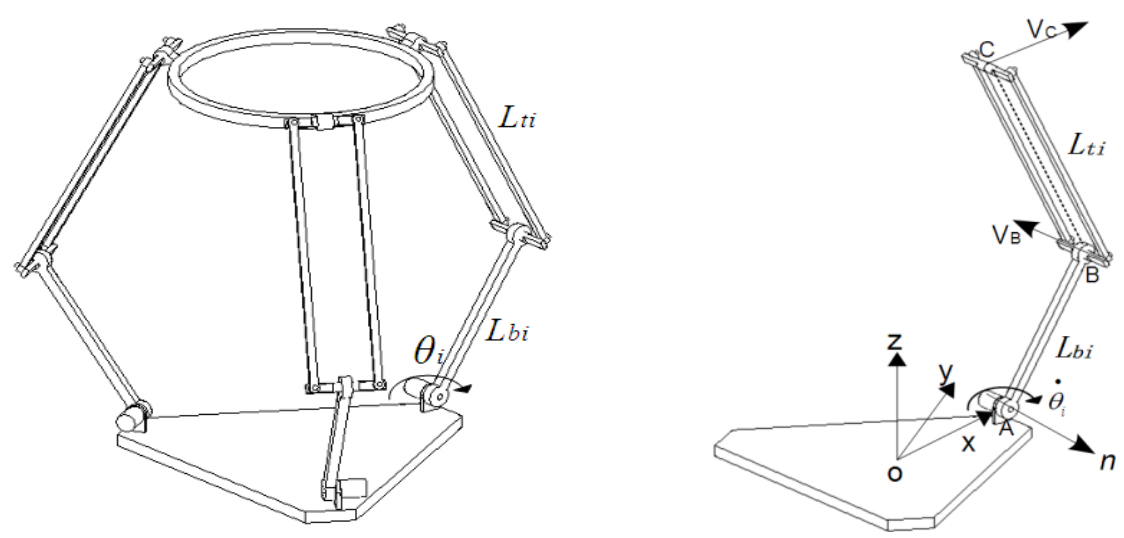

Fig.1 Delta Mechanism

Fig.2 A Branched Chain of Delta Mechanism

\section{Performance Research of Mechanism Based on Jacobi Matrix}

Taking a branched chain of Delta mechanism as shown in Fig.2, at the center of the fixed platform point $\mathrm{O}$ establishes reference coordinate system $\{O X Y Z\}$. Due to the mechanism is only three translational freedoms. The mentioned parallelogram can be represented with virtual link $\boldsymbol{B C}$. $\boldsymbol{V}$ is the speed of the moving platform. Set Parameters of mechanism: vector $\boldsymbol{B}_{i} \boldsymbol{C}_{\boldsymbol{i}}=\boldsymbol{L}_{\boldsymbol{t}}$, vector $\boldsymbol{A}_{i} \boldsymbol{B}_{i}=\boldsymbol{L}_{\boldsymbol{b i}} . \boldsymbol{\Theta}=\left[\begin{array}{lll}\boldsymbol{\theta}_{\boldsymbol{1}} & \boldsymbol{\theta}_{2} & \boldsymbol{\theta}_{3}\end{array}\right]$ as the mechanism input drive angles. $\dot{\theta}_{1}, \dot{\theta}_{2}, \dot{\theta}_{3}$ as velocity size of input angular respectively, the three branched-chain drive on the fixed platform vice uniformly distributed, where: $\boldsymbol{n}_{\boldsymbol{i}}=\left[-\boldsymbol{s i n} \boldsymbol{\alpha}_{\boldsymbol{i}} \boldsymbol{\operatorname { c o s }} \boldsymbol{\alpha}_{\boldsymbol{i}} \boldsymbol{0}\right] . \boldsymbol{\alpha}_{\boldsymbol{i}}$ is the angle between ith branched-chain and the reference coordinate system $\mathrm{X}$ axis, where: $\boldsymbol{\alpha}_{i}=(\mathbf{i}-\mathbf{1}) \cdot \mathbf{2} \boldsymbol{\pi} / \mathbf{3}$. When $\mathrm{i}=1,2,3$, it can be obtain (1).

$$
Q \bullet V=P \bullet \dot{\theta}
$$

$$
\text { Where } Q=\left[\begin{array}{c}
L_{t 1} \\
L_{t 2} \\
L_{t 3}
\end{array}\right] \quad P=\left[\begin{array}{ccc}
L_{t 1} \times n_{1} \bullet L_{b 1} & 0 & 0 \\
0 & L_{t 2} \times n_{2} \bullet L_{b 2} & 0 \\
0 & 0 & L_{t 3} \times n_{3} \bullet L_{b 3}
\end{array}\right]
$$

When the Delta Mechanism is far away from the singularity, from Eqs(1) can be written as (2).

$$
V=J \bullet \dot{\theta}
$$

Where $J=Q^{-1} P$ is the Delta mechanism velocity Jacobian matrix.

\section{The Condition Number Index}

The Jacobin matrix is represented by a linear mapping operation velocity to the joint velocity, the dimensionless Jacobian matrix measures mechanism line dexterity, and the extreme length of vector mapping measures kinematic performance feature characterization of parallel mechanism. This extreme value is defined as the Jacobi singular value, i.e.

$$
k=\sqrt{\lambda\left(J^{T} J\right)}
$$

Use dimensionless Jacobi singular value matrix can be constructed as follows Jacobian matrix condition number:

$$
\xi=k_{\max } / k \min
$$

Where $\kappa_{\text {max }}$ and $\kappa_{\min }$ are the maximum and minimum singular values of $\boldsymbol{J}$. The range of $\xi$ is [1 $\infty]$, in order to tend to the isotropic. $\xi \rightarrow \min$.

When $\xi=1$ the robot has the best performance of the transfer motion, called the shape and 
position of robot for kinematics isotropic. Advantage of condition number method is suitable no matter the Jacobi matrix is square matrix whether or not. Because the Jacobi matrix depend on the position and shape of the robot, so Jacobi matrix condition number is only shows the local property in robot space and only shows the accuracy control information of the robot specific shape and position.

\section{Global Performance Index (GPI)}

According to deficiency performance of Jacobian matrix condition number index, the global condition index [2-3] is introduced to evaluate the global performance of the mechanism in all its workspace as follows:

$$
\eta=\frac{\int_{w} d w / \xi}{\int_{w} d w} \rightarrow \max \quad(0 \leqslant \eta \leqslant 1)
$$

Where $\boldsymbol{w}$ is good-transmission workspace, $\xi$ As the Jacobian matrix condition number of the mechanism in good-transmission workspace. This performance index is based on distribution conditions of Jacobi matrix in the robot whole working space; it is a global performance index. From the definition of the global performance index $\eta$ know that it is the reciprocal of the condition number $1 / \xi$ average in the whole working space, but the performance is not very comprehensive, merely a reflection of the robot in the working space average movement performance. It cannot reflect the performance of the robot in the working space fluctuation range.

\section{Global Performance Volatility Index}

To overcome the deficiency of the global performance index $\eta$, a global motion performance volatility index $\delta$. is proposed as follows:

$$
\delta=\sqrt{\frac{\int_{w}(1 / \xi-\eta)^{2} d w}{\int_{w} d w}}
$$

Where $\delta$ is inverse condition number means variance. Its size can provide the motion performance volatility information of the robot in the whole working space compared to the average level, and $\delta$ can reflect the movement of the robot in the working space stability degree. The new performance $\delta$ can be combined with the global performance index $\eta$ to describe and evaluate mechanism of robot global kinematics and dynamics performance. When the two robot global performance index $\eta$ equal or close, we can according to the fluctuation of performance index $\delta$ to judge and choose.

\section{Optimization Design Considering the Global Index $\eta$ and $\delta$}

A mechanism is not only a better average performance, but also the range of variation is small in the workspace, the stability of mechanism force transfer is better, thus in the robot motion optimization design should be comprehensive consideration the global index $\eta$ and $\delta$. And hope $\eta$ as large as possible and $\delta$ as small as possible, therefore. The optimization objective function is described as follows:

$$
f=\varepsilon \delta-\eta \rightarrow \min
$$

Where $\varepsilon$ as the weight, and $0 \leq \varepsilon$, it is obviously that objective function $f$ is taking into account both the average level the force transmission capability and force transmission capacity in the whole working space of the robot. The larger of the $\varepsilon$ value the force transmission capacity stability of the optimization performance index factors is increasing, work space volume and the motion performance factors reduce, and vice versa. 
Tab.1 Different Values of the Objective Function $\varepsilon$ in the Optimization Results

\begin{tabular}{|l|l|l|l|l|}
\hline $\begin{array}{c}\text { Structure parameters } \\
\varepsilon \text { value }\end{array}$ & $\begin{array}{l}\text { The initiate- } \\
\text { ve arm } l \mathrm{~mm}\end{array}$ & $\begin{array}{l}\text { The driven } \\
\text { arm } l_{t} \mathrm{~mm}\end{array}$ & $\begin{array}{l}\text { Fixed platform } \\
\text { radius } r_{\mathrm{o}} \mathrm{mm}\end{array}$ & $\begin{array}{l}\text { Motion platform } \\
\text { radius } r_{\mathrm{p}} \mathrm{mm}\end{array}$ \\
\hline 1 group $\varepsilon=0.9$ & 240 & 175 & 120 & 80 \\
\hline 2 group $\varepsilon=0.6$ & 250 & 190 & 115 & 50 \\
\hline 3 group $\varepsilon=0.35$ & 170 & 255 & 125 & 60 \\
\hline 4 group $\varepsilon=0$ & 185 & 232 & 128 & 65 \\
\hline
\end{tabular}

Now in the work space (using cylindrical coordinates takes point in the space size of $\pm 120 \times \pm 120 \mathrm{~mm}$, height 150 350mm). The Delta mechanism Parameters is fixed platform radius $r_{\mathrm{o}}$, the moving platform radius $r_{\mathrm{p}}$, the driving arm $l$, driven $\operatorname{arm} l_{t}$. Eqs(7) as the optimization objective function, $\varepsilon=0.9,0.6,0.35,0$, respectively, parameters were optimized ,obviously when $\varepsilon=0$, optimization goal is the global performance index. Through Optimization calculation, the optimization results were obtained see table 1.

Because the $\varepsilon$ size effects the workspace volume weights of optimization objective function, maybe cannot reach the position in a given work space or just is singularity. Therefore, according to the four groups of optimization results to draw a meet point requirements and do not meet the requirements as shown in Fig.3. It is obviously that the group 3 and group 4 all points are meet the requirements From Fig.3, but the two groups pros and cons cannot see from the Fig.3(c, d), we must analyze the performance index from group 3 and group 4.

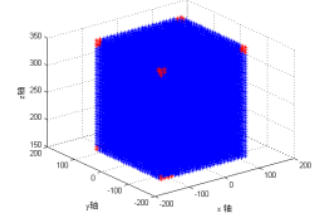

a

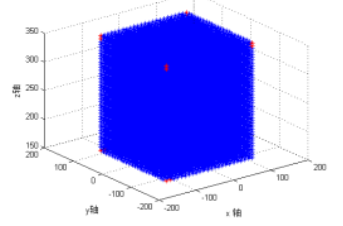

$\mathrm{b}$

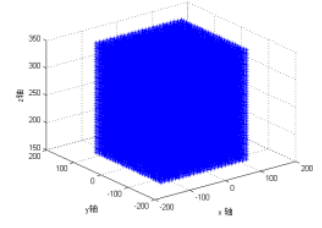

$\mathrm{c}$

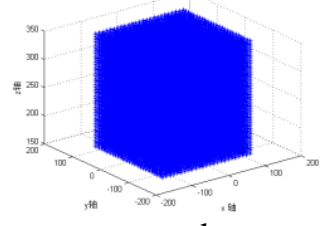

d

Fig. 3 a, b, c, d is respectively Group 1, 2, 3, 4 to Meet the Requirements of Space

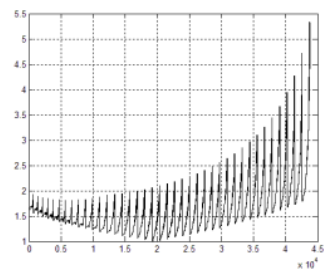

a

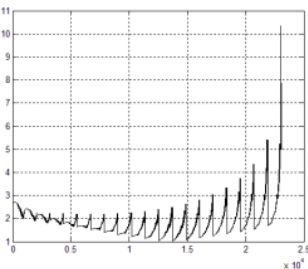

b

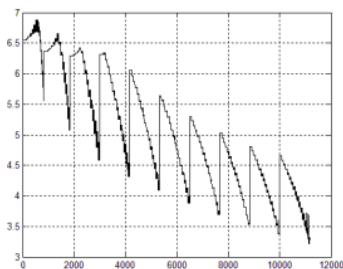

c

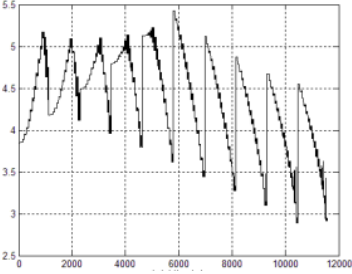

d

Fig.4 a, b is respectively Group 3, 4 the Condition Number Changer; c, d is respectively Group 3, 4 the Force Transfer Changes

3 Groups of global motion performance volatility index $=0.5174$, global performance index $=$ 1.6434 according to Eqs(6), Change the condition number as in Fig.4 a, b.

4 Groups of global motion performance volatility index $=0.6386$, global performance index $=1.8550$ according to Eqs(6), Change the condition number as in Fig.4 a, b.

3 Groups $\eta$ and $\delta$ is obvious smaller, and average condition number is small throughout the given 
space, so the optimization of the 3 group of results is better than that of 4 group.

\section{Global Performance Volatility Index Effects Driving Force}

\section{Static Effect}

Set $T_{F}$ be the driving torque, $\mathrm{F}$ is the working load, in the static case according to the principle of virtual work can be obtained:

$$
T_{F}=J^{T} \cdot F
$$

According to Figs. 4 c, d, the static force transfer and condition number is inverse ratio and the static force transfer has an impact on the Jacobian matrix. Figure $4 \mathrm{c}, \mathrm{d}$ shows that the impact on the volatility index motion performance when condition number is changed. This denotes that group 3 is better than group 4 .

\section{Dynamic Effect}

It can be obtained using the principle of virtual work:

$$
\delta \theta\left(\tau-I_{A} \dot{\theta}-\tau_{A g}\right)-\delta r\left(m_{z} a_{m}+m_{z} g \hat{z}\right)=0
$$

Where $\tau=\left(\begin{array}{lll}\tau_{1} & \tau_{2} & \tau_{3}\end{array}\right)^{T}$ is as active joint torque.

The total mass of the moving platform (including the movable platform $(1.2 \mathrm{~kg})$, load mass, The $1 / 3$ equivalent mass of driven arm), $a_{m}=2$ as the moving platform the center of mass acceleration, $\mathrm{I}_{\mathrm{A}}$ For the active arm is equivalent to the shaft rotational inertia, where $\mathrm{I}_{\mathrm{A}}=\mathrm{I}_{\mathrm{b}}+\mathrm{I}^{2} \mathrm{I}_{\mathrm{j}}+2 \mathrm{l}^{2}{ }_{\mathrm{b}} \mathrm{m}_{\mathrm{t}} / 3, \mathrm{~m}_{\mathrm{t}}=0.21 \mathrm{~kg}$ as the driven arm mass, $\mathrm{I}_{\mathrm{b}}=0.03273 \mathrm{~kg} \cdot \mathrm{m}^{2}$ as the active arm is equivalent to the shaft rotational inertia, $\mathrm{i}=86$ as the reducer gear ratio, $\mathrm{I}_{\mathrm{j}}=7 \times 10^{-7}$ for the moment of inertia of the reducer, $\tau_{A g}$ as heavy moment of driving arm shaft, $\hat{z}$ as coordinate the corresponding $0-x y z$ axis of the unit vector.

Substituting $l_{b i} \delta \theta=J \delta r$ to $\operatorname{Eqs}(8)$ obtained:

$$
\begin{aligned}
& \tau=I_{A} \ddot{\theta}_{+} \tau_{A g}+\left(m_{z} a_{m}+m_{z} g \hat{z}\right) J^{-T}=I_{A}\left(J a_{m} / l_{b i}+c(V) / l_{b i}^{2}\right)+\left(m_{z} a_{m}+m_{z} g \hat{z}\right) J^{-T}+\tau_{A g}= \\
& I_{A}\left(J / l_{b i}+m_{z} J^{-T}\right) a_{m}+I_{A} c(v) / l_{b i}^{2}+m_{z} g \hat{z} J^{-T}+\tau_{A g}=\tau_{A}+\tau_{V}+\tau_{g}
\end{aligned}
$$

Where $c(V)=\left(\begin{array}{lll}c_{1}^{T}(V) & c_{2}^{T}(V) & \left.c_{3}^{T}(V)\right)^{T}\end{array}\right.$

Where

$$
c_{i}(V)=V^{T}\left(\left(\frac{L_{t i}\left(n_{i} \times L_{b i}\right)^{T}\left(n_{i} \times L_{b i}\right) L_{t i}}{\left(n_{i}^{T}\left(L_{b i} \times L_{t i}\right)\right)^{3}}+\frac{l_{b i}}{l_{t i} n_{i}^{T}\left(L_{b i} \times L_{t i}\right)}\left(E_{3}-\frac{2 L_{t i}\left(n_{i} \times L_{b i}\right)^{T}}{n_{i}^{T}\left(L_{b i} \times L_{t i}\right)}\right)\right.\right.
$$

$$
\left.\left.+\frac{L_{t i} L_{t i}^{T}}{\left(n_{i}^{T}\left(L_{b i} \times L_{t i}\right)\right)^{2}}\right)\right) V
$$

Eqs(9) including three parts:

$$
\tau_{A}=\left(I_{A} J / l_{b i}+m_{z} l_{b i} J^{-T}\right) a_{m}, \tau_{V}=I_{A} c(V) / l_{b i}^{2}, \tau_{g}=m_{z} g \hat{z} J^{-T}+\tau_{A g}
$$

Hypothesis $a_{m}$ as a constant, the global performance volatility index which influence to $\tau_{A}$ with the change of condition number as shown in Fig.6.

$$
m_{z}=1.515 \mathrm{~kg}, l_{b i}=0.125 \mathrm{~m}, 0.128 \mathrm{~m}, I_{A}=0.03877 \mathrm{~kg} \cdot \mathrm{m}^{2}, 0.03951 \mathrm{~kg} \cdot \mathrm{m}^{2}, a_{m}=2 \mathrm{~m} / \mathrm{s}^{2}
$$




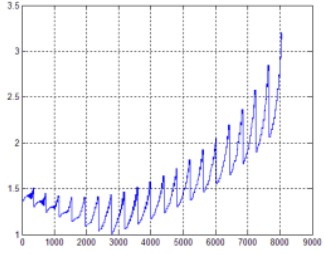

a

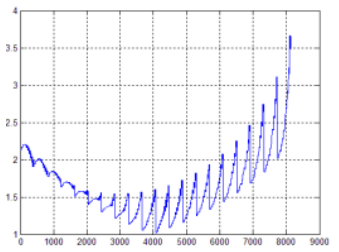

b

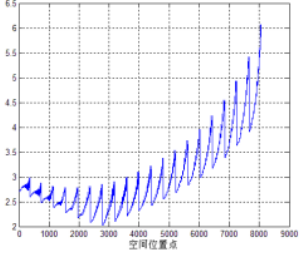

c

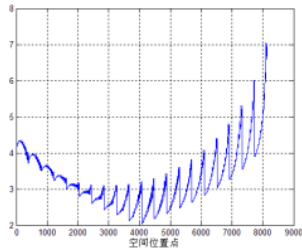

d

Fig. 6 a, b is respectively Group 3, 4 Global Performance Volatility Index Effect to $\tau_{A}$; c, d is respectively Group 3, 4 Global Performance Volatility Index Effect to $\tau_{g}$

The global performance volatility indexes almost no influence to $\tau_{V}=I_{A} c(V) / l_{b i}^{2}$. The global performance volatility index which influence to $\tau_{g}=m_{z} g \hat{z} J^{-T}+\tau_{A g}$ with the change of condition number as shown in Fig.6 c, d.

From the dynamic partial analysis, it is understood that the impact on four group four is apparent than group four. According to Fig. 6 c, d, it is believed that group three works more efficient than group four. If several groups of the optimization results are same or similar, it can use the fluctuation of performance index to judge the superiority or not.

\section{Conclusion}

This paper suggests a new index, so called the global performance volatility index that is applied to measure the $\delta$ which can reflect the movement of the robot in the working space stability degree. The following conclusions are drawn: (1) According to Jacobin matrix, analyzes the condition index and the global performance index to overcoming their shortcomings, this paper puts forward motion performance fluctuation index. (2) Optimization function was established according to the fluctuation index of global motion performance, and the mechanism was optimized and satisfactory results were obtained. (3) Analysis and comprise the static transfer ability and Dynamics effect the global motion performance fluctuation index degree. It is a good basis to design and application.

\section{Acknowledgement}

The work reported in this paper is funded by Jiangsu's university brand Specialty construction projects funded projects (Grant No. PPZY2015C232). Address: Nanjing Polytechnic Institute, No.625, Geguan Road, Dachang, Luhe District, Nanjing City, Jiangsu Province, P.R.China. Zip code: 210048.

\section{References}

[1] J.K.Salisbury, J. Craig: "Force Control and Kinematic Issues"[J]. Int. J of Robot. Res, 1982, 1(1):4 17.

[2] Gosselin C, Angeles J. A global performance index for the kinematic optimization of robotic manipulators [J]. ASME Journal of Mechanical Design. 1991. 11(3):220 226.

[3] Gosselin C, Angeles J. A New Performance Index for the Kinematic Optimization of Robotic Manipulators [J]. 20th Proc. Biennial Mechanisms Conference [C]. Kissimmee, FL, 1988. 441 447

[4] C. Gosselin, J. Angeles, The optimum kinematic design of a spherical three Degree-of-Freedom parallel manipulator, Journal of Mechanisms, Transmissions and Automation in Design, Trans. ASME ,1989,111 (2): 202-207.

[5] X. Jun Liu. Optimum design of 3-DOF spherical parallel manipulators with respect to the conditioning and stiffness indices [J], Mechanism and Machine Theory, 2000, 35 (9):1257-1267. 
[6] L.A. Silva, J.M. Sebastian, R. Saltaren, etal. RoboTenis: optimal design of a parallel robot with high performance [J], 2005 IEEE/RSJ International Conference on Intelligent Robots and Systems, 2005. 2134-2139.

[7] Lung-Wen Tsai, Gregory C. Kinematics of a Novel Three DOF Translational Platform [J], Proceedings of the 1996 IEEE International Conference on Robotics and Automation Minneapolis, Minnesota - April 1996, 4:3446 - 3451.

[8] ZHAO Jie, ZHU Yan-He CAI He-gao Geometric solution for direct kinematics of delta parallel robot [J]. Journal of Harbin Institue of Technology, 2003, 35 (1):25-27.

[9] Lung-Wen Tsai, Sameer Joshi. Kinematics of 3- DOF Position Mechanisms for Use in Hybrid Kinematic Machines [J], Journal of mechanical Design. June 2002, Vol 124:245-253.

[10] Bi Shusheng, Zong Guanghua. Analysis of Kinematics of Delta Parallel Micromanipulator with Vector Space Method [J]. Journal of Beijing University of Aeronautics and Astronautics. 2003, 29(4): $3446-3451$. 